\title{
Distribution, wealth and demand regimes in historical perspective: the USA, the UK, France and Germany, 1855-2010
}

\section{Online appendices}

\author{
Engelbert Stockhammer* \\ King's College London, UK \\ Joel Rabinovich \\ University of Leeds, UK \\ Niall Reddy \\ New York University, NY, USA
}


2 Review of Keynesian Economics, Vol. 9 No. 3

\section{APPENDIX 1}

Table A1 Descriptive statistics, all variables in logarithms

\begin{tabular}{lccrrr}
\hline \multicolumn{7}{c}{ USA } \\
\hline \multicolumn{1}{c}{$C$} & $I$ & \multicolumn{1}{c}{$Y$} & \multicolumn{1}{c}{$P W$} & $W S$ \\
\hline Mean & 7.947636 & 6.686643 & 8.365639 & 9.584874 & -0.271346 \\
Median & 7.991780 & 6.861958 & 8.457950 & 9.608397 & -0.268862 \\
Maximum & 9.244649 & 7.992656 & 9.593592 & 11.066460 & -0.167533 \\
Minimum & 6.471595 & 3.841740 & 6.668695 & 8.207497 & -0.341473 \\
Std dev. & 0.843327 & 0.980194 & 0.850252 & 0.847959 & 0.030870 \\
Skewness & -0.095350 & -0.904181 & -0.319308 & 0.057983 & 0.264485 \\
Kurtosis & 1.818409 & 3.317431 & 2.055704 & 1.865008 & 3.869143 \\
Observations & 81 & 81 & 81 & 81 & 81
\end{tabular}

\begin{tabular}{lccccr}
\hline \multicolumn{7}{c}{ UK } \\
\hline \multicolumn{1}{c}{$C$} & $I$ & $Y$ & $P W$ & $W S$ \\
\hline Mean & 5.343059 & 3.608282 & 5.609543 & 7.187709 & -0.334816 \\
Median & 5.208009 & 3.287440 & 5.497372 & 7.090669 & -0.315528 \\
Maximum & 6.873047 & 5.622481 & 7.224690 & 8.875994 & -0.147879 \\
Minimum & 3.906704 & 1.478108 & 4.035734 & 6.039404 & -0.491946 \\
Std dev. & 0.785356 & 1.180058 & 0.854481 & 0.676355 & 0.085634 \\
Skewness & 0.246137 & 0.160247 & 0.165835 & 0.804391 & -0.042405 \\
Kurtosis & 2.148374 & 1.680134 & 2.008606 & 3.186301 & 1.970331 \\
Observations & 152 & 152 & 152 & 152 & 152 \\
& & & & &
\end{tabular}

\begin{tabular}{|c|c|c|c|c|c|}
\hline \multicolumn{6}{|c|}{ France } \\
\hline & $C$ & $I$ & $Y$ & $P W$ & $W S$ \\
\hline Mean & 5.766576 & 4.516263 & 6.167922 & 7.432209 & -0.283410 \\
\hline Median & 5.515481 & 4.538228 & 6.016874 & 7.038061 & -0.270073 \\
\hline Maximum & 7.024829 & 6.064757 & 7.580520 & 9.187811 & -0.158659 \\
\hline Minimum & 4.783939 & 3.143084 & 4.987498 & 6.152613 & -0.507961 \\
\hline Std dev. & 0.778016 & 1.032412 & 0.932482 & 0.843499 & 0.064903 \\
\hline Skewness & 0.282198 & -0.004172 & 0.168741 & 0.512601 & -0.882630 \\
\hline Kurtosis & 1.480761 & 1.313962 & 1.389808 & 2.009366 & 4.355678 \\
\hline Observations & 104 & 104 & 104 & 104 & 104 \\
\hline \multicolumn{6}{|c|}{ Germany } \\
\hline & $C$ & $I$ & $Y$ & $P W$ & $W S$ \\
\hline Mean & 5.715155 & 4.572698 & 6.057340 & 7.341663 & -0.285989 \\
\hline Median & 5.621093 & 4.716108 & 6.050761 & 7.019845 & -0.282835 \\
\hline Maximum & 7.288286 & 6.221515 & 7.701440 & 9.116346 & -0.197927 \\
\hline Minimum & 4.022335 & 2.261534 & 4.254475 & 6.185562 & -0.419318 \\
\hline Std dev. & 1.075236 & 1.281311 & 1.155995 & 0.927571 & 0.047222 \\
\hline Skewness & 0.035204 & -0.144308 & -0.014998 & 0.524827 & -0.378499 \\
\hline Kurtosis & 1.498085 & 1.405322 & 1.435003 & 1.807959 & 2.754600 \\
\hline Observations & 115 & 115 & 115 & 115 & 115 \\
\hline
\end{tabular}




\section{APPENDIX 2}

\section{Table A2 Unit-root tests}

\begin{tabular}{|c|c|c|c|c|c|c|c|}
\hline \multicolumn{8}{|c|}{ USA } \\
\hline & $Y$ & $P W$ & $W S$ & $C$ & $C$ & I corp. & $L T R$ \\
\hline $\begin{array}{l}\mathrm{ADF} \\
p \text { value } \\
\text { Unit root }\end{array}$ & $\begin{array}{c}-0.911792 \\
0.9490 \\
\text { Yes } \\
d Y\end{array}$ & $\begin{array}{c}-0.114225 \\
0.9938 \\
\text { Yes } \\
d P W\end{array}$ & $\begin{array}{c}-2.092 \\
0.2484 \\
\text { Yes } \\
d W S\end{array}$ & $\begin{array}{c}-0.495984 \\
0.9818 \\
\text { Yes } \\
d C\end{array}$ & $\begin{array}{c}-3.063 \\
0.1238 \\
\text { Yes } \\
d I\end{array}$ & $\begin{array}{c}-3.161 \\
0.1020 \\
\text { Yes } \\
\text { dIcorp }\end{array}$ & $\begin{array}{c}-2.248 \\
0.4555 \\
\text { Yes }\end{array}$ \\
\hline $\begin{array}{l}\mathrm{ADF} \\
p \text { value } \\
\text { Unit root }\end{array}$ & $\begin{array}{c}-6.353 \\
0.0000 \\
\text { No }\end{array}$ & $\begin{array}{c}-6.652 \\
0.0000 \\
\text { No }\end{array}$ & $\begin{array}{c}-6.044 \\
0.0000 \\
\text { No }\end{array}$ & $\begin{array}{c}-4.393 \\
0.0039 \\
\text { No }\end{array}$ & $\begin{array}{c}-5.676 \\
0.0001 \\
\mathrm{No}\end{array}$ & $\begin{array}{c}-5.514 \\
0.0001 \\
\text { No }\end{array}$ & \\
\hline \multicolumn{8}{|c|}{ UK } \\
\hline & $Y$ & $P W$ & $W S$ & $C$ & $C$ & $L T R$ & \\
\hline $\begin{array}{l}\text { ADF } \\
p \text { value } \\
\text { Unit root }\end{array}$ & $\begin{array}{c}1.858 \\
1.000 \\
\text { Yes } \\
d Y\end{array}$ & $\begin{array}{c}3.058 \\
1.000 \\
\text { Yes } \\
d P W\end{array}$ & $\begin{array}{c}-2.680 \\
0.2464 \\
\text { Yes } \\
d W S\end{array}$ & $\begin{array}{c}1.477 \\
1.000 \\
\text { Yes } \\
d C\end{array}$ & $\begin{array}{c}-1.254 \\
0.8948 \\
\text { Yes } \\
d I\end{array}$ & $\begin{array}{c}-4.931 \\
0.0005 \\
\text { No }\end{array}$ & \\
\hline $\begin{array}{l}\mathrm{ADF} \\
p \text { value } \\
\text { Unit root }\end{array}$ & $\begin{array}{c}-4.850 \\
0.0006 \\
\text { No }\end{array}$ & $\begin{array}{c}-6.111 \\
0.0000 \\
\text { No }\end{array}$ & $\begin{array}{c}-7.661 \\
0.0000 \\
\text { No }\end{array}$ & $\begin{array}{c}-4.885 \\
0.0005 \\
\text { No }\end{array}$ & $\begin{array}{c}-8.270 \\
0.0000 \\
\text { No }\end{array}$ & & \\
\hline \multicolumn{8}{|c|}{ France } \\
\hline & $Y$ & $P W$ & $W S$ & $C$ & $C$ & I corp. & $L T R$ \\
\hline $\begin{array}{l}\text { ADF } \\
p \text { value } \\
\text { Unit root }\end{array}$ & $\begin{array}{c}-1.214 \\
0.9024 \\
\text { Yes } \\
d Y \\
\end{array}$ & $\begin{array}{c}3.921 \\
1.000 \\
\text { Yes } \\
d P W \\
\end{array}$ & $\begin{array}{c}-2.985 \\
0.1410 \\
\text { Yes } \\
d W S \\
\end{array}$ & $\begin{array}{c}-1.673 \\
0.7561 \\
\text { Yes } \\
d C \\
\end{array}$ & $\begin{array}{c}-1.721 \\
0.7357 \\
\text { Yes } \\
d I \\
\end{array}$ & $\begin{array}{c}-1.674 \\
0.7562 \\
\text { Yes } \\
\text { dIcorp } \\
\end{array}$ & $\begin{array}{c}-3.117 \\
0.1075 \\
\text { Yes }\end{array}$ \\
\hline $\begin{array}{l}\mathrm{ADF} \\
p \text { value } \\
\text { Unit root }\end{array}$ & $\begin{array}{c}-4.311 \\
0.0044 \\
\text { No }\end{array}$ & $\begin{array}{c}-2.352 \\
0.0187 \\
\text { No }\end{array}$ & $\begin{array}{c}-6.854 \\
0.0000 \\
\text { No }\end{array}$ & $\begin{array}{c}-5.011 \\
0.0004 \\
\text { No }\end{array}$ & $\begin{array}{c}-6.419 \\
0.0000 \\
\text { No }\end{array}$ & $\begin{array}{c}-6.585 \\
0.0000 \\
\text { No }\end{array}$ & \\
\hline \multicolumn{8}{|c|}{ Germany } \\
\hline & $Y$ & $P W$ & $W S$ & $C$ & $C$ & $L T R$ & \\
\hline $\begin{array}{l}\mathrm{ADF} \\
p \text { value } \\
\text { Unit root }\end{array}$ & $\begin{array}{c}-1.587 \\
0.7928 \\
\text { Yes } \\
d Y\end{array}$ & $\begin{array}{c}4.168 \\
1.000 \\
\text { Yes } \\
d P W\end{array}$ & $\begin{array}{c}-3.447 \\
0.0502 \\
\text { No } \\
d W S\end{array}$ & $\begin{array}{c}-1.084 \\
0.9264 \\
\text { Yes } \\
d C\end{array}$ & $\begin{array}{c}-2.532 \\
0.3123 \\
\text { Yes } \\
d I\end{array}$ & $\begin{array}{c}-3.447 \\
0.0502 \\
\text { No }\end{array}$ & \\
\hline $\begin{array}{l}\text { ADF } \\
p \text { value } \\
\text { Unit root }\end{array}$ & $\begin{array}{c}-9.708 \\
0.0000 \\
\text { No }\end{array}$ & $\begin{array}{c}-9.500 \\
0.0000 \\
\text { No }\end{array}$ & $\begin{array}{c}-6.937 \\
0.0000 \\
\text { No }\end{array}$ & $\begin{array}{c}-5.110 \\
0.0003 \\
\text { No }\end{array}$ & $\begin{array}{c}-6.474 \\
0.0000 \\
\text { No }\end{array}$ & & \\
\hline
\end{tabular}


4 Review of Keynesian Economics, Vol. 9 No. 3

\section{APPENDIX 3}

Table A3 Regression results for consumption shares in differences, USA, UK, France and Germany

\begin{tabular}{|c|c|c|c|c|c|c|c|c|}
\hline & \multicolumn{2}{|c|}{$\mathrm{USA}^{\mathrm{a}}$} & \multicolumn{2}{|c|}{$\mathrm{UK}^{\mathrm{b}}$} & \multicolumn{2}{|c|}{ France $^{c}$} & \multicolumn{2}{|c|}{ Germany $^{\mathrm{d}}$} \\
\hline & 1 & 2 & 1 & 2 & 1 & 2 & 1 & 2 \\
\hline$\Delta w s$ & 0.599 & 0.403 & 0.049 & 0.089 & 0.055 & 0.131 & 0.463 & 0.444 \\
\hline$t$-stat & 4.374 & 2.703 & 0.793 & 1.304 & 0.764 & 1.850 & 4.667 & 4.096 \\
\hline$\Delta p w / y$ & 0.293 & 0.355 & 0.224 & 0.245 & -0.164 & -0.104 & 0.422 & 0.438 \\
\hline$t$-stat & 5.048 & 6.025 & 5.659 & 5.944 & -2.497 & -1.567 & 7.387 & 7.058 \\
\hline$\Delta c / y(-1)$ & 0.355 & 0.486 & 0.200 & 0.132 & -0.258 & -0.322 & 0.297 & 0.323 \\
\hline$t$-stat & 3.327 & 4.289 & 3.651 & 2.164 & -2.759 & -3.599 & 3.150 & 3.108 \\
\hline$\Delta w s(-1)$ & 0.167 & 0.229 & 0.041 & 0.075 & 0.115 & 0.113 & 0.060 & 0.054 \\
\hline$t$-stat & 1.075 & 1.382 & 0.654 & 1.083 & 1.550 & 1.567 & 0.549 & 0.477 \\
\hline$\Delta p w / y(-1)$ & -0.089 & -0.191 & -0.057 & -0.064 & 0.193 & 0.209 & -0.161 & -0.198 \\
\hline$t$-stat & -1.305 & -2.669 & -1.425 & -1.344 & 2.797 & 2.927 & -2.681 & -2.496 \\
\hline$\Delta c / y(-2)$ & - & -0.290 & - & 0.179 & - & -0.022 & - & -0.163 \\
\hline$t$-stat & - & -2.505 & - & 2.369 & - & -0.251 & - & -1.625 \\
\hline$\Delta w s(-2)$ & - & -0.129 & - & 0.012 & - & 0.333 & - & 0.169 \\
\hline$t$-stat & - & -0.850 & - & 0.204 & - & 4.189 & - & 1.501 \\
\hline$\Delta p w / y(-2)$ & - & 0.216 & - & 0.014 & - & -0.091 & - & 0.067 \\
\hline$t$-stat & - & 2.872 & - & 0.333 & - & -1.346 & - & 1.029 \\
\hline obs & 80 & 79 & 154 & 153 & 102 & 100 & 114 & 111 \\
\hline$R^{2}$ & 0.752 & 0.785 & 0.782 & 0.796 & 0.380 & 0.491 & 0.615 & 0.620 \\
\hline DW & 1.914 & 2.163 & 2.368 & 2.295 & 2.024 & 1.752 & 1.784 & 1.800 \\
\hline BG serial correl. & 0.523 & 0.009 & 0.023 & 0.001 & 0.672 & 0.296 & 0.059 & 0.077 \\
\hline \multicolumn{9}{|l|}{ Long-run effects } \\
\hline$w s$ & 1.188 & 0.626 & 0.113 & 0.254 & 0.135 & 0.429 & 0.743 & 0.795 \\
\hline$p w$ & 0.316 & 0.474 & 0.209 & 0.282 & 0.023 & 0.011 & 0.371 & 0.364 \\
\hline
\end{tabular}

Notes: a. Estimation period 1929-2010. Controlling for significant war years (1942, 1943, 1944, 1945).

b. Estimation period 1855-2010. Controlling for significant crisis and war years $(1914,1915,1916,1917$, 1918, 1919, 1921, 1939, 1940, 1941, 1942, 1943, 1944, 1945).

c. Estimation period 1896-2010. Controlling for significant crisis and war years (1903, 1910, 1917, 1932). Original database has no information for consumption during WWII years.

d. Estimation period 1869-2010. Controlling for significant reunification years (1990). Original database has no information for consumption during WWI, hyperinflation and WWII years. 


\section{APPENDIX 4}

Table A4 Marginal effects of consumption, total investment and corporate investment to WS, $P W$ and $Y$, calculated at the mean of the sample

\begin{tabular}{lcrrc}
\hline Consumption & Preferred specification vs specification 3 & $W S$ & $P W$ & $Y$ \\
\hline USA & 2 & 0.503 & 0.089 & 0.434 \\
& 3 & 1.575 & 0.102 & 0.391 \\
UK & 1 & 0.716 & 0.052 & 0.518 \\
& 3 & 1.574 & 0.085 & 0.385 \\
France & 1 & -0.443 & 0.013 & 0.576 \\
& 3 & 0.052 & -0.002 & 0.585 \\
Germany & 1 & 0.262 & 0.022 & 0.581 \\
& 3 & -0.015 & -0.015 & 0.629
\end{tabular}

\begin{tabular}{lcrrc}
\hline Investment & Preferred specification vs specification 3 & \multicolumn{1}{c}{$W S$} & \multicolumn{1}{c}{$P W$} & $Y$ \\
\hline USA & 1 & 0.204 & -0.028 & 0.334 \\
& 3 & -0.911 & -0.010 & 0.209 \\
UK & 1 & 0.033 & -0.021 & 0.220 \\
& 3 & 0.018 & -0.023 & 0.226 \\
France & 1 & 0.124 & 0.003 & 0.210 \\
& 3 & 0.297 & -0.001 & 0.213 \\
Germany & 2 & 0.543 & 0.075 & 0.168 \\
& 3 & 0.786 & -0.006 & 0.059 \\
& & & & \\
\hline Corporate investment & Preferred specification vs specification 3 & $W S$ & $P W$ & $Y$ \\
\hline USA & 1 & -0.218 & -0.056 & 0.344 \\
& 3 & -0.087 & -0.031 & 0.234 \\
France & 1 & -0.113 & 0.007 & 0.089 \\
& 3 & -0.037 & 0.007 & 0.086 \\
\hline
\end{tabular}


6 Review of Keynesian Economics, Vol. 9 No. 3

\section{APPENDIX 5}

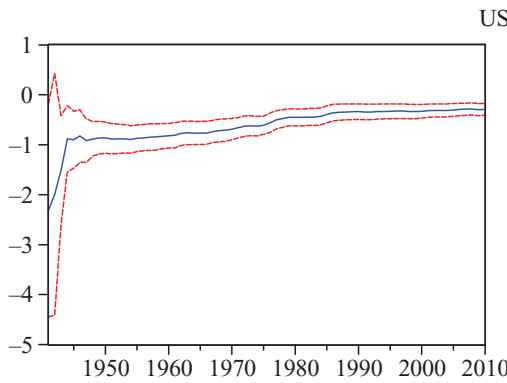

USA
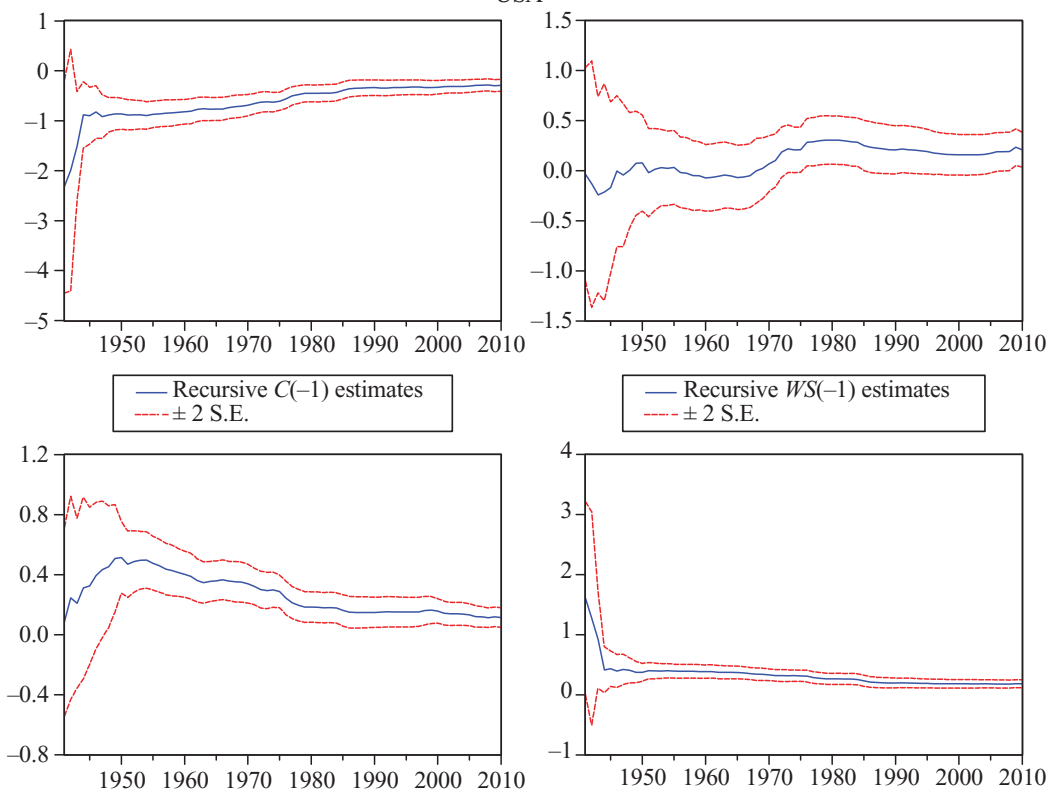

- Recursive $P W(-1)$ estimates $-\ldots 2$ S.E.

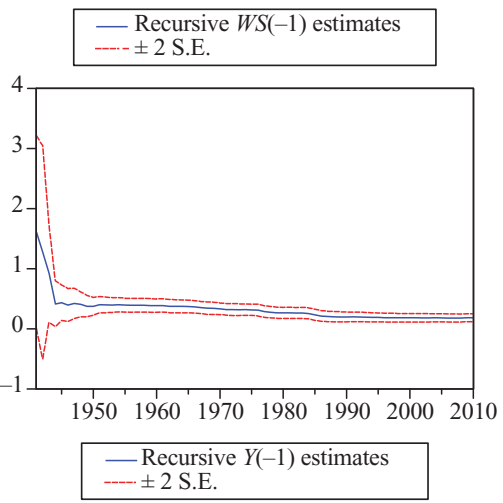

UK
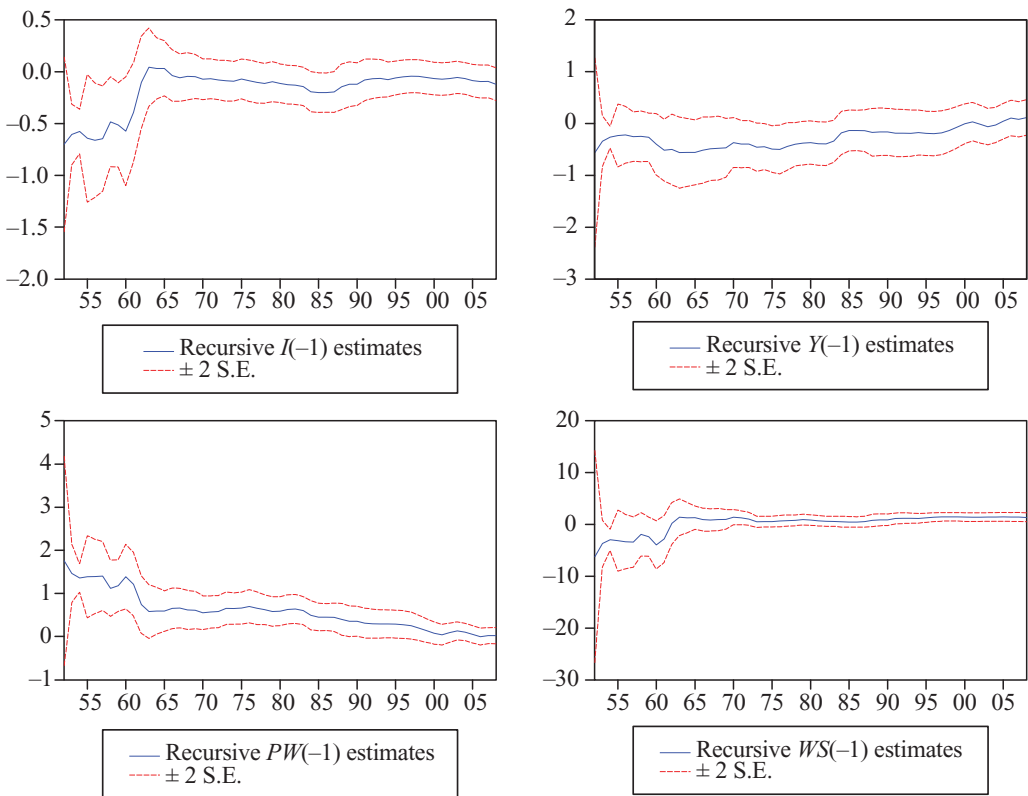

Figure A1 Recursive estimations for consumption parameters, USA and UK 

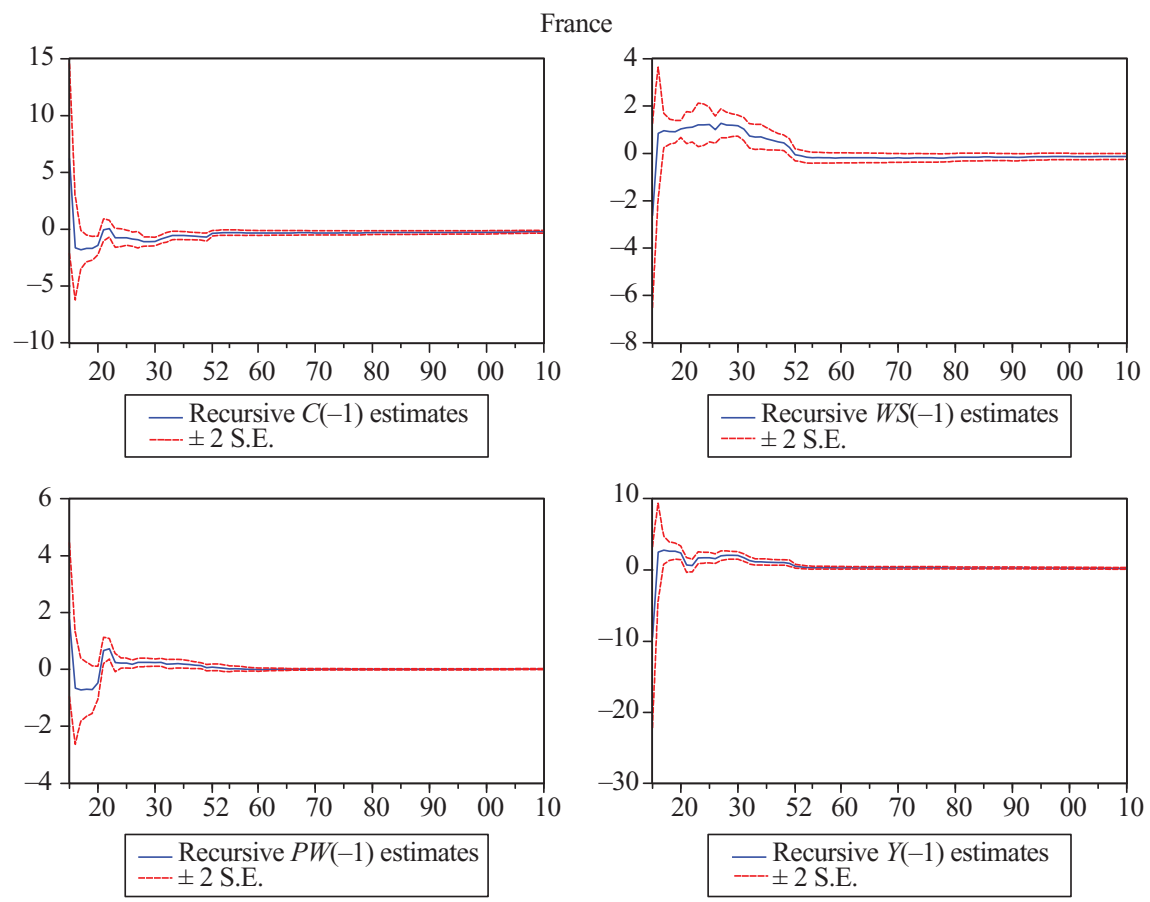

Germany
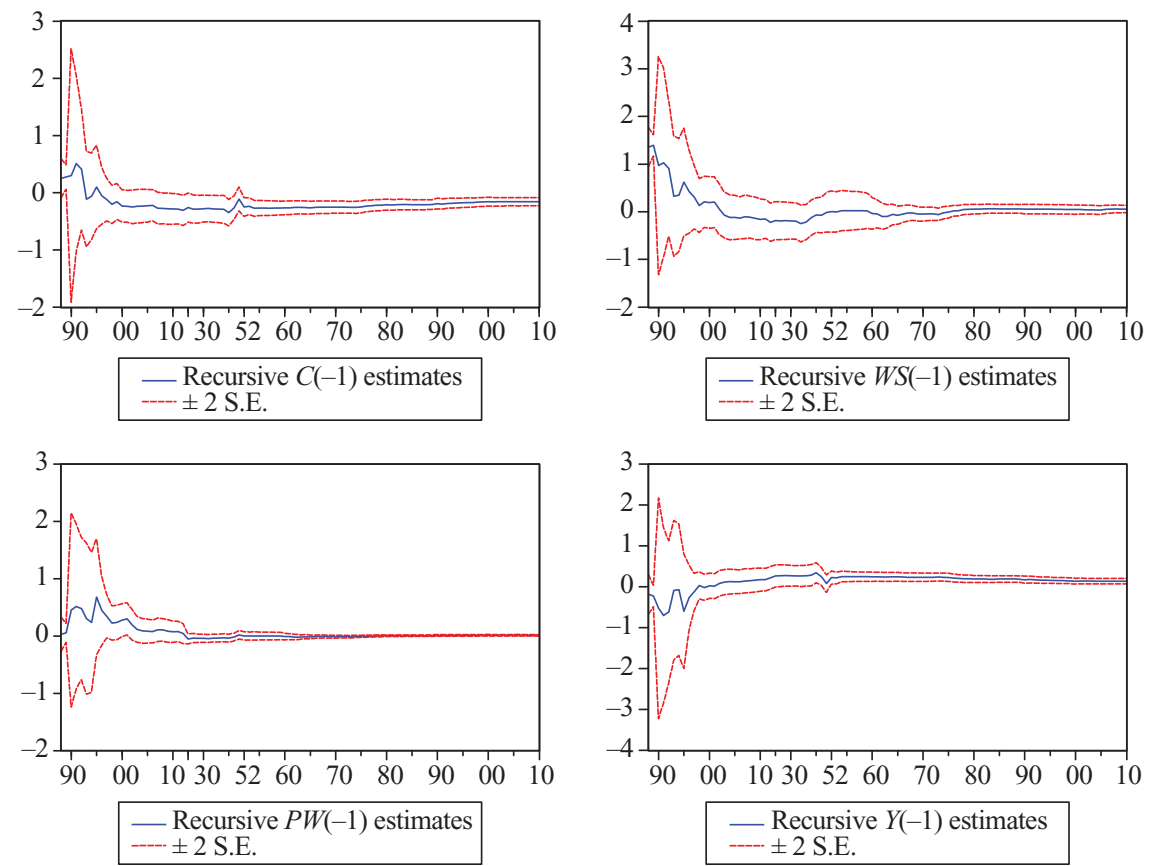

Figure A2 Recursive estimations for consumption parameters, France and Germany 
8 Review of Keynesian Economics, Vol. 9 No. 3

USA
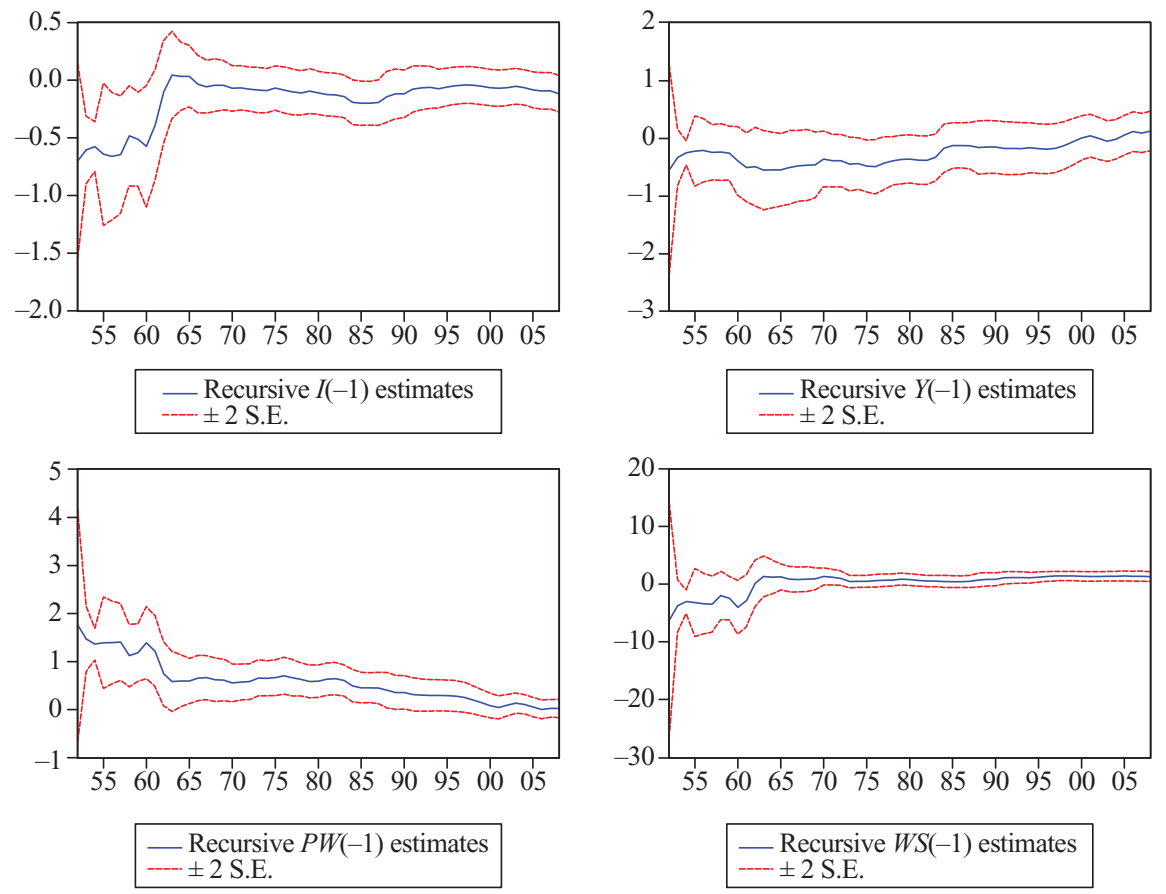

UK
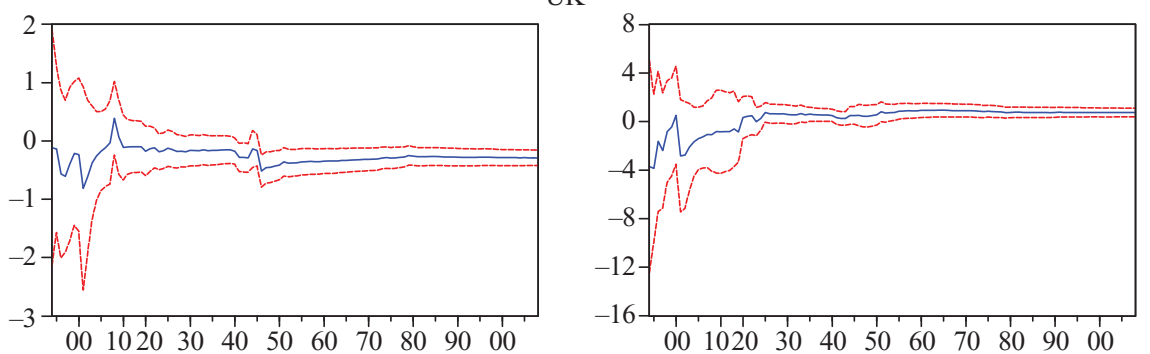

$\begin{array}{llllllllllll}00 & 1020 & 30 & 40 & 50 & 60 & 70 & 80 & 90 & 00\end{array}$

- Recursive $I(-1)$ estimates \pm 2 S.E.

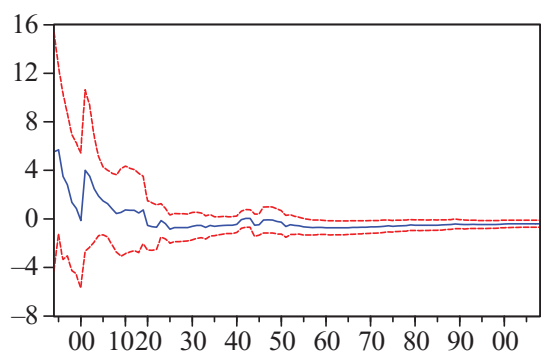

- Recursive $Y(-1)$ estimates $-\cdots \pm 2$ S.E.

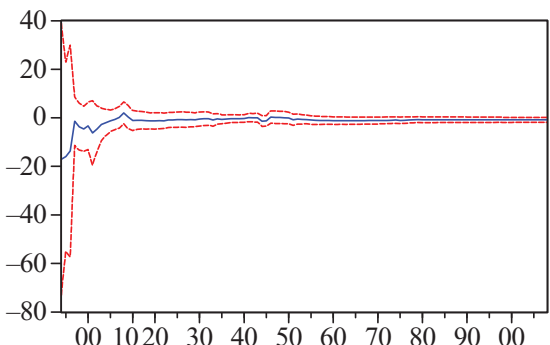

$\begin{array}{lllllllllllll}00 & 1020 & 30 & 40 & 50 & 60 & 70 & 80 & 90 & 00\end{array}$

— Recursive $P W(-1)$ estimates

- Recursive WS(-1) estimates \pm 2 S.E.

Figure A3 Recursive estimations for investment parameters, USA and UK 
France
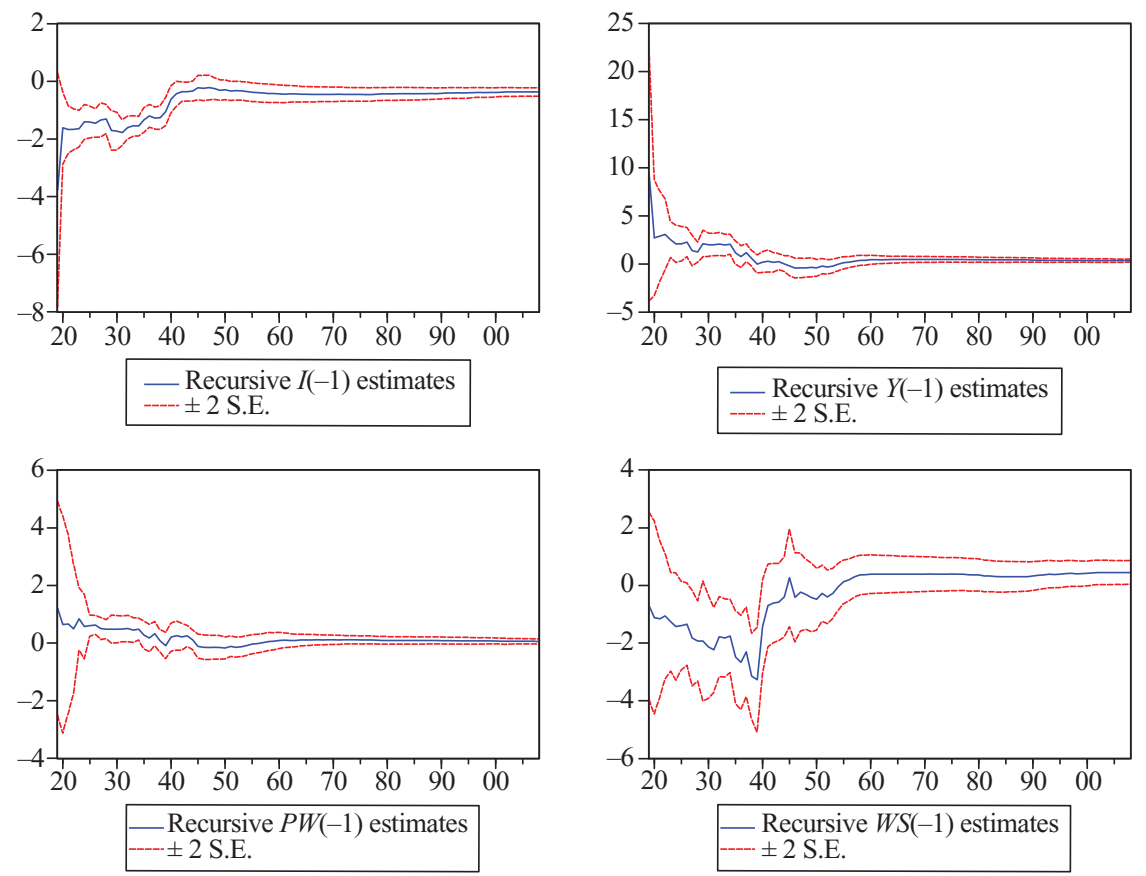

Germany
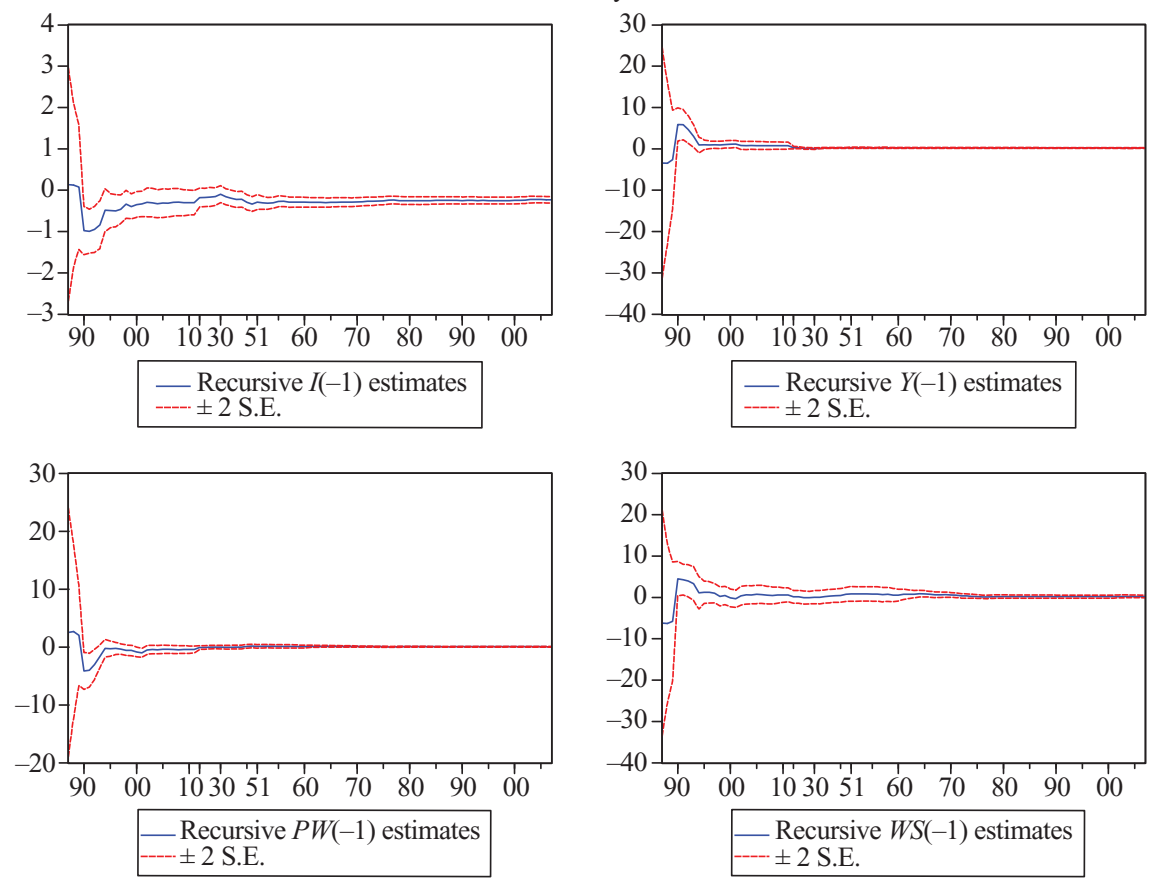

Figure A4 Recursive estimations for investment parameters, France and Germany 
10 Review of Keynesian Economics, Vol. 9 No. 3

USA
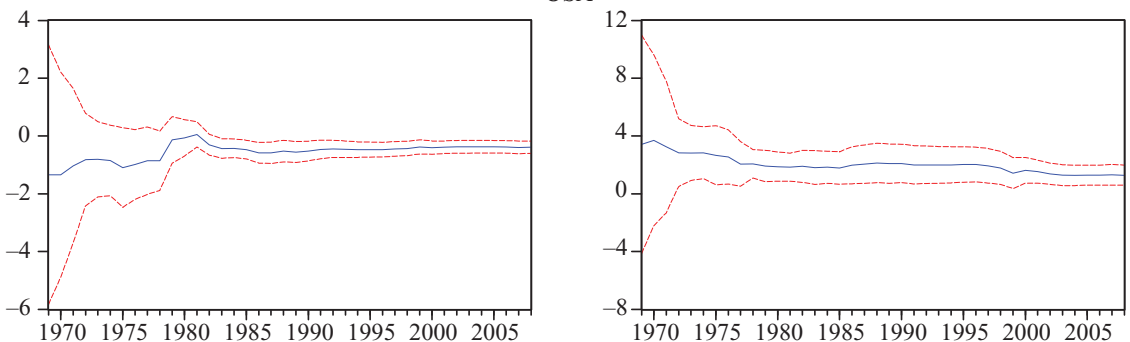

- Recursive I CORP $(-1)$ estimates \pm 2 S.E.
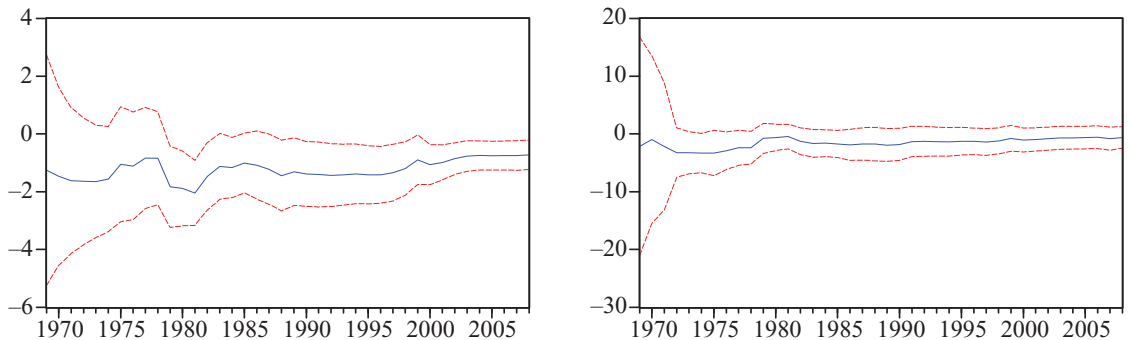

- Recursive $P W(-1)$ estimates

- Recursive $W S(-1)$ estimates \pm 2 S.E.

France
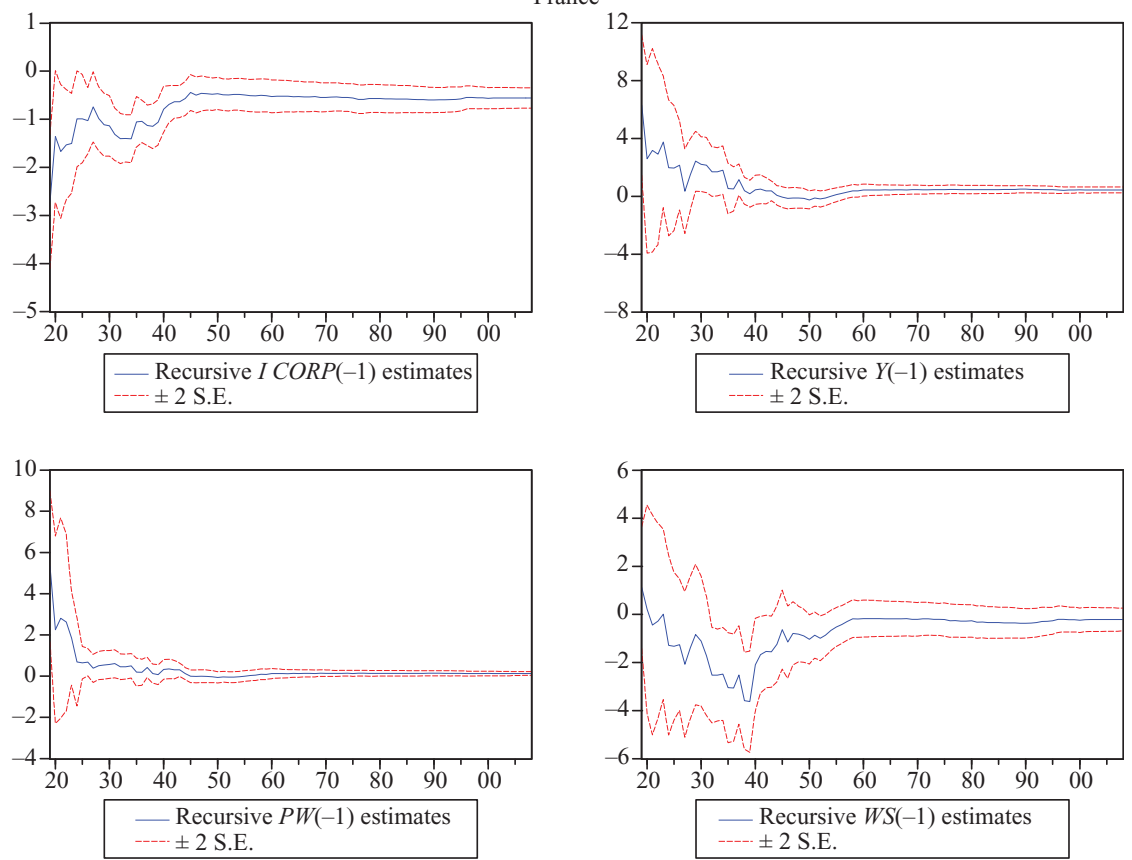

Figure A5 Recursive estimations for corporate investment parameters, USA and France 


\section{APPENDIX 6}

Table A5 Sub-samples estimation for consumption equations, France, Germany and $U K$

\begin{tabular}{|c|c|c|c|c|c|c|}
\hline & \multicolumn{2}{|c|}{ France $^{\mathrm{a}}$} & \multicolumn{2}{|c|}{ Germany $^{b}$} & \multicolumn{2}{|c|}{$\mathrm{UK}^{\mathrm{c}}$} \\
\hline & 1896-1946 & 1946-2010 & 1869-1946 & 1946-2010 & 1855-1946 & 1946-2010 \\
\hline$c(-1)$ & -0.708 & -0.162 & -0.111 & 0.042 & -0.377 & -0.179 \\
\hline$t$-stat & -3.838 & -1.465 & -1.059 & 0.616 & -5.605 & -3.050 \\
\hline$w s(-1)$ & 0.250 & 0.048 & -0.012 & 0.049 & 0.219 & 0.152 \\
\hline$t$-stat & 1.387 & 0.884 & -0.060 & 1.103 & 3.156 & 2.789 \\
\hline$p w(-1)$ & 0.055 & 0.008 & 0.017 & 0.002 & 0.037 & 0.104 \\
\hline$t$-stat & 1.001 & 0.603 & 0.481 & 0.072 & 1.447 & 3.888 \\
\hline$y(-1)$ & 0.917 & 0.139 & 0.079 & -0.056 & 0.313 & 0.038 \\
\hline$t$-stat & 4.071 & 1.302 & 0.744 & -1.028 & 4.718 & 0.797 \\
\hline$\Delta w s$ & 0.157 & 0.134 & 0.332 & 0.361 & 0.076 & 0.031 \\
\hline$t$-stat & 1.004 & 1.157 & 1.864 & 2.940 & 0.654 & 0.372 \\
\hline$\Delta p w$ & 0.115 & -0.007 & 0.043 & 0.147 & 0.111 & 0.258 \\
\hline$t$-stat & 0.529 & -0.102 & 0.298 & 1.238 & 1.133 & 6.566 \\
\hline$\Delta y$ & 1.235 & 0.696 & 0.459 & 0.625 & 0.701 & 0.506 \\
\hline$t$-stat & 7.359 & 5.664 & 5.022 & 7.066 & 8.959 & 6.310 \\
\hline$\Delta c(-1)$ & -0.063 & -0.019 & 0.148 & -0.183 & -0.006 & 0.122 \\
\hline$t$-stat & -0.324 & -0.104 & 0.846 & -1.325 & -0.072 & 1.129 \\
\hline$\Delta w s(-1)$ & -0.260 & -0.014 & 0.074 & 0.059 & -0.069 & -0.157 \\
\hline$t$-stat & -1.800 & -0.138 & 0.369 & 0.414 & -0.580 & -1.936 \\
\hline$\Delta p w(-1)$ & -0.394 & 0.089 & -0.191 & -0.141 & 0.017 & -0.018 \\
\hline$t$-stat & -1.261 & 0.894 & -1.490 & -1.012 & 0.131 & -0.344 \\
\hline$\Delta y(-1)$ & -0.184 & -0.039 & 0.082 & 0.193 & 0.005 & -0.115 \\
\hline$t$-stat & -0.730 & -0.181 & 0.649 & 1.296 & 0.054 & -1.122 \\
\hline$\Delta c(-2)$ & 0.289 & 0.011 & -0.402 & -0.195 & 0.018 & -0.028 \\
\hline$t$-stat & 1.666 & 0.061 & -2.425 & -1.525 & 0.165 & -0.273 \\
\hline$\Delta w s(-2)$ & -0.023 & -0.046 & 0.176 & 0.229 & -0.146 & 0.019 \\
\hline$t$-stat & -0.151 & -0.523 & 0.901 & 1.919 & -1.351 & 0.251 \\
\hline$\Delta p w(-2)$ & -0.371 & -0.061 & 0.151 & -0.018 & -0.071 & 0.038 \\
\hline$t$-stat & -1.234 & -0.670 & 1.514 & -0.173 & -0.737 & 0.778 \\
\hline$\Delta y(-2)$ & -0.521 & -0.077 & 0.157 & 0.193 & -0.055 & 0.016 \\
\hline$t$-stat & -2.242 & -0.388 & 1.360 & 1.554 & -0.517 & 0.160 \\
\hline obs & 41 & 59 & 52 & 59 & 89 & 65 \\
\hline$R^{2}$ & 0.910 & 0.831 & 0.781 & 0.896 & 0.927 & 0.889 \\
\hline DW & 1.924 & 2.001 & 2.181 & 2.069 & 2.218 & 2.021 \\
\hline BG serial correl. & 0.7851 & 0.5630 & 0.1063 & 0.8470 & 0.3826 & 0.6780 \\
\hline \multicolumn{7}{|l|}{ Long-run effects } \\
\hline ws & 0.353 & 0.297 & -0.112 & -1.163 & 0.580 & 0.848 \\
\hline$p w$ & 0.078 & 0.051 & 0.155 & -0.052 & 0.099 & 0.582 \\
\hline$y$ & 1.296 & 0.858 & 0.716 & 1.325 & 0.831 & 0.211 \\
\hline
\end{tabular}

Notes: a. Dummies for 1903, 1910, 1917 and 1932. Data for 1940-1948 are missing.

b. Dummy for 1990. Data for 1914-1924 and 1939-1949 are missing.

c. Dummies for 1914, 1915, 1916, 1917, 1918, 1919, 1921, 1939, 1940, 1941, 1942, 1943, 1944, 1945 and 1955. 
12 Review of Keynesian Economics, Vol. 9 No. 3

Table A6 Sub-samples estimation for investment equations, France, Germany and UK

\begin{tabular}{|c|c|c|c|c|c|c|}
\hline & \multicolumn{2}{|c|}{ France $^{\mathrm{a}}$} & \multicolumn{2}{|c|}{ Germany $^{b}$} & \multicolumn{2}{|c|}{$\mathrm{UK}^{\mathrm{c}}$} \\
\hline & 1896-1946 & 1946-2010 & 1869-1946 & $1946-2010$ & 1855-1946 & 1946-2010 \\
\hline$i(-1)$ & -0.753 & -0.325 & -0.270 & -0.234 & -0.172 & -0.645 \\
\hline$t$-stat & -3.967 & -5.733 & -2.270 & -2.830 & -1.822 & -4.579 \\
\hline$y(-1)$ & 1.048 & 0.181 & 0.212 & 0.538 & 0.775 & 1.228 \\
\hline$t$-stat & 1.810 & 1.699 & 1.807 & 2.962 & 3.654 & 3.980 \\
\hline$p w(-1)$ & 0.258 & 0.124 & 0.109 & -0.204 & -0.676 & -0.411 \\
\hline$t$-stat & 1.160 & 1.979 & 0.610 & -2.175 & -2.417 & -2.642 \\
\hline$w s(-1)$ & -0.013 & 0.239 & 0.717 & -0.212 & -0.550 & 0.793 \\
\hline$t$-stat & -0.025 & 1.330 & 0.840 & -1.079 & -0.870 & 2.129 \\
\hline $\operatorname{LTR}(-1)$ & -0.041 & 0.578 & -3.285 & -0.298 & -3.554 & 0.853 \\
\hline$t$-stat & -0.102 & 7.740 & -3.240 & -0.466 & -3.451 & 1.862 \\
\hline$\Delta y$ & 0.501 & 2.111 & 2.977 & 3.330 & 1.711 & 3.295 \\
\hline$t$-stat & 1.154 & 7.161 & 9.970 & 9.279 & 2.228 & 5.452 \\
\hline$\Delta p w$ & -0.256 & -0.175 & 0.686 & -0.171 & -0.087 & -0.271 \\
\hline$t$-stat & -0.396 & -0.719 & 0.979 & -0.376 & -0.160 & -0.980 \\
\hline$\Delta w s$ & -0.206 & -0.483 & 0.929 & 0.953 & 0.676 & 1.552 \\
\hline$t$-stat & -0.572 & -1.242 & 1.238 & 2.126 & 0.535 & 2.381 \\
\hline$\Delta L T R$ & 0.563 & 0.124 & -2.790 & -0.681 & -1.665 & 0.547 \\
\hline$t$-stat & 2.007 & 1.278 & -3.544 & -1.437 & -2.044 & 1.217 \\
\hline$\Delta i(-1)$ & 0.538 & -0.257 & 0.108 & 0.124 & -0.301 & 0.232 \\
\hline$t$-stat & 2.253 & -3.251 & 0.751 & 0.786 & -2.334 & 3.933 \\
\hline$\Delta y(-1)$ & 0.525 & 1.125 & -0.213 & -1.484 & -0.479 & -0.293 \\
\hline$t$-stat & 0.987 & 3.485 & -0.366 & -2.538 & -0.591 & -0.455 \\
\hline$\Delta p w(-1)$ & 1.591 & 0.887 & -1.119 & -0.032 & 0.414 & 0.413 \\
\hline$t$-stat & 1.611 & 2.687 & -1.421 & -0.057 & 0.441 & 1.130 \\
\hline$\Delta w s(-1)$ & 0.755 & -0.814 & 0.851 & -0.021 & -1.913 & 0.168 \\
\hline$t$-stat & 1.985 & -2.329 & 1.119 & -0.042 & -1.447 & 0.250 \\
\hline$\Delta \operatorname{LTR}(-1)$ & 0.166 & -0.065 & 0.621 & 0.016 & -0.292 & -1.124 \\
\hline$t$-stat & 0.480 & -0.590 & 0.855 & 0.043 & -0.598 & -3.015 \\
\hline$\Delta i(-2)$ & 0.555 & -0.209 & 0.161 & 0.193 & -0.298 & -0.003 \\
\hline$t$-stat & 2.250 & -2.569 & 1.565 & 1.216 & -2.332 & -0.048 \\
\hline$\Delta y(-2)$ & -0.045 & 1.072 & -0.317 & -0.725 & 0.666 & 0.019 \\
\hline$t$-stat & -0.096 & 4.223 & -0.554 & -1.231 & 0.948 & 0.030 \\
\hline$\Delta p w(-2)$ & -1.041 & 0.082 & -0.346 & 0.749 & 1.682 & 0.125 \\
\hline$t$-stat & -1.030 & 0.269 & -0.464 & 1.732 & 2.074 & 0.376 \\
\hline$\Delta w s(-2)$ & 0.404 & 0.287 & 0.073 & 0.067 & -0.279 & -0.068 \\
\hline$t$-stat & 1.040 & 0.832 & 0.123 & 0.169 & -0.228 & -0.131 \\
\hline$d L T R(-2)$ & 0.366 & 0.060 & 0.596 & 0.218 & 0.302 & -0.350 \\
\hline$t$-stat & 1.381 & 0.768 & 0.997 & 0.854 & 0.655 & -0.982 \\
\hline obs & 48 & 63 & 49 & 56 & 68 & 62 \\
\hline$R^{2}$ & 0.962 & 0.899 & 0.977 & 0.891 & 0.913 & 0.851 \\
\hline DW & 2.078 & 1.999 & 2.327 & 1.947 & 2.071 & 2.041 \\
\hline BG serial correl. & 0.8538 & 0.5838 & 0.3925 & 0.7791 & 0.8528 & 0.6256 \\
\hline \multicolumn{7}{|l|}{ Long-run effects } \\
\hline$w s$ & -0.018 & 0.736 & 2.659 & -0.906 & -3.206 & 1.229 \\
\hline$p w$ & 0.342 & 0.383 & 0.406 & -0.874 & -3.944 & -0.637 \\
\hline$y$ & 1.393 & 0.556 & 0.787 & 2.301 & 4.518 & 1.903 \\
\hline
\end{tabular}

Notes: a. Dummies for 1919, 1925, 1930, 1936, 1938, 1939, 1940, 1941, 1942, 1943, 1945, 1976 and 1993. b. Dummies for 1930, 1931, 1932, 1933 and 1990. Data for 1914-1919 and 1939-1945 are missing. Depreciation information starts in 1925; we computed a constant rate of depreciation before.

c. Dummies for 1876, 1880, 1908, 1940, 1941, 1942, 1943, 1944, 1945, 1946, 1950 and 1951. 


\section{APPENDIX 7}

Table A7 Sub-samples estimation for consumption equations, USA, UK, France and Germany, 1946-1980 and 1980-2010

\begin{tabular}{|c|c|c|c|c|c|c|c|c|}
\hline & \multicolumn{2}{|c|}{ USA } & \multicolumn{2}{|c|}{$\mathrm{UK}^{\mathrm{a}}$} & \multicolumn{2}{|c|}{ France } & \multicolumn{2}{|c|}{ Germany $^{b}$} \\
\hline & $\begin{array}{c}1946- \\
1980\end{array}$ & $\begin{array}{c}1980- \\
2010\end{array}$ & $\begin{array}{c}1946- \\
1980\end{array}$ & $\begin{array}{c}1980- \\
2010\end{array}$ & $\begin{array}{c}1946- \\
1980\end{array}$ & $\begin{array}{c}1980- \\
2010\end{array}$ & $\begin{array}{c}1946- \\
1980\end{array}$ & $\begin{array}{c}1980- \\
2010\end{array}$ \\
\hline-1$)$ & -0.516 & 113 & -0.418 & -0.249 & -0.784 & -0.475 & -0.118 & -0.694 \\
\hline & 5.104 & 2 & -3.151 & -1.394 & -2.333 & -2.559 & -0.506 & -2.279 \\
\hline$w s(-1)$ & -0.079 & 0.108 & 0.180 & -0.017 & 0.106 & 0.027 & 0.354 & 0.640 \\
\hline$t$-stat & -0.449 & 0.540 & 0.705 & -0.144 & 0.828 & 0.273 & 1.707 & .740 \\
\hline$p w(-1)$ & -0.094 & 0.247 & 0.064 & 0.130 & -0.065 & 0.027 & -0.006 & .115 \\
\hline$t$-stat & -1.777 & 1.602 & 1.782 & 1.769 & -0.896 & 1.213 & -0.139 & .084 \\
\hline$y(-1)$ & 0.604 & -0.458 & 0.268 & 0.020 & 0.792 & 0.390 & 0.086 & 0.692 \\
\hline$t$-stat & 5.349 & -0.838 & 2.288 & 0.220 & 2.319 & 2.263 & 0.402 & 2.451 \\
\hline$\Delta w s$ & -0.250 & 0.3 & 0.230 & 7 & 0.042 & 0.202 & .256 & .519 \\
\hline$t$-stat & -1.333 & 2 & 1.267 & 0.693 & 0.206 & 1.199 & .816 & 2.612 \\
\hline$\Delta p w$ & 0.005 & 7 & 0.140 & 0.200 & 0.025 & 0.058 & 0.121 & -0.142 \\
\hline & 0.065 & 65 & 2.041 & 309 & 0.099 & 0.777 & 0.716 & -0.377 \\
\hline$\Delta y$ & 0.442 & 0.856 & 0.604 & 0.748 & 0.725 & 0.659 & 0.580 & 0.784 \\
\hline t) & 4.660 & 5.075 & 4.005 & 5.625 & 3.272 & 3.602 & 3.736 & 4.176 \\
\hline$\Delta c(-1$ & 0.008 & -0.5 & .076 & -0.383 & 0.348 & -0.189 & 0.318 & .134 \\
\hline 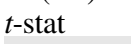 & 0.054 & 1.630 & .388 & -1.652 & 1.043 & -0.694 & -1.286 & 0.370 \\
\hline$\Delta w s$ & -0.223 & 0.320 & .195 & 0.183 & -0.008 & -0.147 & -0.131 & -0.079 \\
\hline stat & -1.167 & 1.373 & -1.557 & 1.382 & -0.045 & -0.984 & -0.444 & -0.242 \\
\hline$\Delta p w(-1)$ & 0.195 & -0.229 & 0.158 & -0.122 & 0.214 & 0.031 & -0.201 & 0.213 \\
\hline & 488 & -1.9 & & -1.694 & 0.881 & 0.319 & -1.009 & 0.583 \\
\hline$\Delta y$ & -0.301 & & 0.088 & & -0.319 & -0.171 & 0.184 & -0.295 \\
\hline$t$-st & -3.161 & & .450 & & -0.853 & -0.641 & 0.717 & -0.715 \\
\hline$\Delta c(-2)$ & -0.091 & -0.369 & 0.083 & -0.297 & 0.055 & 0.187 & -0.223 & 0.565 \\
\hline$t$-stat & -0.724 & -1.382 & 0.576 & -1.405 & 0.173 & 0.785 & -0.926 & 1.628 \\
\hline$\Delta w s$ & -0.111 & & 0.048 & & 0.001 & -0.189 & 027 & -0.769 \\
\hline & -07 & & 6 & 9 & 0.006 & -1 & .112 & -2.081 \\
\hline$\Delta p w(-2$ & -0.092 & -0.199 & -0.056 & -0.004 & -0.228 & -0.023 & .113 & -0.179 \\
\hline$t$-stat & -0.874 & -1.993 & -0.640 & -0.047 & -0.862 & -0.222 & 0.715 & -0.583 \\
\hline$\Delta y(-2)$ & 0.080 & 0.550 & -0.160 & 0.305 & -0.109 & -0.144 & 0.097 & -0.705 \\
\hline$t$-stat & 0.763 & 1.981 & -0.965 & 1.388 & -0.314 & -0.614 & 0.550 & -1.777 \\
\hline obs & 34 & 31 & 34 & 31 & 29 & 21 & 29 & 31 \\
\hline$R^{2}$ & 0.892 & & 0.907 & 0.96 & 0.846 & 0.826 & 0.908 & 0.853 \\
\hline DW & 1.169 & 1.694 & 1.969 & 2.109 & 2.063 & 2.307 & 2.470 & 2.269 \\
\hline \multicolumn{9}{|l|}{ Long-ru } \\
\hline$w s$ & 0.154 & 55 & -0.432 & 67 & -0.135 & -0.057 & -2.997 & -0.922 \\
\hline$p w$ & 0.182 & & -0.154 & -0.522 & 0.083 & -0.057 & 0.048 & -0.165 \\
\hline$y$ & -1.170 & -4.050 & -0.642 & -0.081 & -1.011 & -0.821 & -0.725 & -0.996 \\
\hline
\end{tabular}

Notes: a. Dummy for 1955.

b. Dummy for 1990 . 
14 Review of Keynesian Economics, Vol. 9 No. 3

Table A8 Sub-samples estimation for investment equations, USA, UK, France and Germany, 1946-1980 and 1980-2010

\begin{tabular}{|c|c|c|c|c|c|c|c|c|}
\hline & \multicolumn{2}{|c|}{ USA } & \multicolumn{2}{|c|}{$\mathrm{UK}^{\mathrm{a}}$} & \multicolumn{2}{|c|}{ France $^{\mathrm{b}}$} & \multicolumn{2}{|c|}{ Germany $^{c}$} \\
\hline & $\begin{array}{c}1946- \\
1980\end{array}$ & $\begin{array}{c}1980- \\
2010\end{array}$ & $\begin{array}{c}1946- \\
1980\end{array}$ & $\begin{array}{c}1980- \\
2010\end{array}$ & $\begin{array}{c}1946- \\
1980\end{array}$ & $\begin{array}{c}1980- \\
2010\end{array}$ & $\begin{array}{c}1946- \\
1980\end{array}$ & $\begin{array}{c}1980- \\
2010\end{array}$ \\
\hline$i(-1)$ & -0.263 & -0.389 & -1.223 & 0.406 & -0.822 & -0.456 & -0.582 & -0.075 \\
\hline$t$-stat & -1.309 & -2.330 & -7.502 & 0.979 & -1.870 & -1.951 & -2.606 & -0.127 \\
\hline$y(-1)$ & 0.735 & -1.034 & 2.701 & -0.209 & 0.209 & -0.264 & 0.970 & 1.413 \\
\hline$t$-stat & 1.490 & -1.196 & 4.027 & -0.308 & 0.413 & -0.780 & 2.955 & 0.972 \\
\hline$p w(-1)$ & -0.486 & 0.927 & -0.620 & -0.047 & 0.513 & 0.385 & -0.178 & -0.940 \\
\hline$t$-stat & -1.232 & 1.490 & -1.918 & -0.179 & 1.330 & 1.676 & -1.622 & -2.217 \\
\hline$w s(-1)$ & 0.325 & -0.775 & -0.417 & 0.027 & -0.747 & -0.626 & -2.448 & -1.392 \\
\hline$t$-stat & 0.519 & -0.399 & -0.261 & 0.026 & -1.055 & -0.718 & -2.345 & -1.422 \\
\hline $\operatorname{LTR}(-1)$ & -0.085 & -2.750 & -0.091 & -0.540 & 0.411 & -1.382 & -2.500 & 0.123 \\
\hline$t$-stat & -0.067 & -2.116 & -0.096 & -0.476 & 0.984 & -0.794 & -1.945 & 0.056 \\
\hline$\Delta y$ & 3.182 & 3.355 & 4.539 & 0.737 & 1.070 & 4.054 & 2.300 & 4.054 \\
\hline$t$-stat & 11.400 & 4.912 & 4.308 & 0.725 & 1.186 & 4.784 & 3.656 & 7.069 \\
\hline$\Delta p w$ & 0.171 & -0.058 & -0.167 & 0.991 & -0.114 & 0.062 & -1.102 & 0.628 \\
\hline$t$-stat & 0.425 & -0.170 & -0.251 & 2.889 & -0.124 & 0.147 & -1.687 & 0.557 \\
\hline$\Delta w s$ & -0.119 & -0.973 & -0.107 & -2.089 & -1.616 & 0.257 & -2.053 & 1.349 \\
\hline$t$-stat & -0.179 & -0.616 & -0.081 & -1.882 & -1.732 & 0.453 & -1.639 & 2.333 \\
\hline$\Delta L T R$ & -0.067 & -1.189 & 2.080 & 0.580 & -0.182 & -0.076 & -0.299 & 0.069 \\
\hline$t$-stat & -0.124 & -0.868 & 2.424 & 0.880 & -0.571 & -0.076 & -0.396 & 0.087 \\
\hline$\Delta i(-1)$ & -0.457 & -0.292 & 0.168 & -0.792 & -0.264 & -0.388 & 0.429 & -0.657 \\
\hline$t$-stat & -1.910 & -1.132 & 1.415 & -1.396 & -0.977 & -1.483 & 1.700 & -1.422 \\
\hline$\Delta y(-1)$ & 1.033 & 2.629 & 0.265 & 0.725 & 0.709 & 1.779 & -1.527 & 0.651 \\
\hline$t$-stat & 1.411 & 2.179 & 0.232 & 0.627 & 1.172 & 1.335 & -2.102 & 0.451 \\
\hline$\Delta p w(-1)$ & 0.118 & -0.348 & -1.320 & 0.524 & -0.073 & -0.265 & 0.384 & 1.539 \\
\hline$t$-stat & 0.438 & -0.551 & -1.445 & 1.476 & -0.099 & -0.513 & 0.603 & 1.521 \\
\hline$\Delta w s(-1)$ & 0.651 & 0.695 & 2.786 & -1.743 & -0.454 & 0.053 & -0.003 & 0.969 \\
\hline$t$-stat & 0.927 & 0.519 & 1.636 & -2.142 & -0.718 & 0.063 & -0.003 & 1.548 \\
\hline$\Delta L T R(-1)$ & 0.254 & 0.723 & 0.622 & 0.000 & 0.050 & 1.956 & 1.749 & -0.740 \\
\hline$t$-stat & 0.447 & 0.758 & 0.803 & 0.000 & 0.173 & 2.267 & 2.307 & -0.678 \\
\hline$\Delta \mathrm{i}(-2)$ & 0.034 & -0.184 & 0.245 & -0.596 & -0.212 & -0.216 & 0.142 & -0.440 \\
\hline$t$-stat & 0.188 & -0.802 & 2.163 & -1.602 & -1.150 & -0.971 & 0.650 & -1.479 \\
\hline$\Delta y(-2)$ & -1.439 & 1.388 & 0.127 & 1.042 & 1.486 & 1.556 & 0.043 & -0.534 \\
\hline$t$-stat & -2.331 & 1.265 & 0.113 & 0.843 & 3.055 & 0.979 & 0.057 & -0.439 \\
\hline$\Delta p w(-2)$ & 1.043 & -1.015 & 1.158 & 1.038 & -0.470 & 0.152 & 0.269 & 0.122 \\
\hline$t$-stat & 2.945 & -2.532 & 1.248 & 2.614 & -0.707 & 0.332 & 0.487 & 0.104 \\
\hline$\Delta w s(-2)$ & -0.465 & 0.070 & 1.259 & -0.832 & 0.827 & 0.007 & 0.040 & 0.940 \\
\hline$t$-stat & -0.852 & 0.050 & 1.188 & -0.884 & 1.148 & 0.011 & 0.053 & 1.841 \\
\hline$d L T R(-2)$ & 0.083 & -0.024 & 0.165 & 0.368 & 0.161 & 0.634 & 1.253 & 0.047 \\
\hline$t$-stat & 0.262 & -0.036 & 0.279 & 0.564 & 0.879 & 0.744 & 2.373 & 0.082 \\
\hline obs & 34 & 29 & 34 & 29 & 34 & 29 & 29 & 28 \\
\hline$R^{2}$ & 0.972 & 0.968 & 0.970 & 0.946 & 0.905 & 0.950 & 0.974 & 0.975 \\
\hline DW & 2.687 & 2.514 & 2.960 & 2.658 & 1.945 & 2.182 & 2.635 & 2.715 \\
\hline \multicolumn{9}{|c|}{ Long-run effects } \\
\hline$w s$ & -1.239 & 1.991 & 0.341 & 0.068 & 0.909 & 1.372 & 4.209 & 18.539 \\
\hline$p w$ & 1.850 & -2.382 & 0.507 & -0.116 & -0.624 & -0.844 & 0.306 & 12.525 \\
\hline$y$ & -2.799 & 2.658 & -2.208 & -0.516 & -0.254 & 0.579 & -1.668 & -18.819 \\
\hline
\end{tabular}

Notes: a. Dummies for 1950 and 1951.

b. Dummies for 1976 and 1993.

c. Dummy for 1990. 